\title{
Early Prediction of the Carbapenem Resistance Gram-negative Bacteria Carriage in Intensive Care Unit using Machine-Learning
}

\author{
Qiqiang Liang \\ Zhejiang University School of Medicine Second Affiliated Hospital \\ Qinyu Zhao \\ Healsci technology co., Itd \\ Xin Xu \\ Zhejiang University School of Medicine Second Affiliated Hospital \\ Yu Zhou \\ Zhejiang University School of Medicine second Affiliated Hospital \\ Man Huang ( $\nabla$ huangman@zju.edu.cn) \\ Zhejiang University School of Medicine https://orcid.org/0000-0002-0842-2254
}

\section{Research}

Keywords: Machine learning, Carbapenem resistance gram-negative bacteria, Multi-drug resistant bacteria prediction, multivariate logistic regression, Prevention and control of nosocomial infection

Posted Date: August 25th, 2020

DOI: https://doi.org/10.21203/rs.3.rs-60222/v1

License: (c) (i) This work is licensed under a Creative Commons Attribution 4.0 International License. Read Full License 


\section{Abstract \\ Background}

The prevention and control of carbapenem-resistance gram-negative bacteria (CR-GNB) is the difficulty and focus for clinicians in the intensive care unit (ICU). This study construct a CR-GNB carriage prediction model in order to predict the CR-GNB incidence in one week.

\section{Methods}

The database is comprised of nearly 10,000 patients. the model is constructed by the multivariate logistic regression model and three machine learning algorithms. Then we choose the optimal model and verify the accuracy by daily predicted and recorded the occurrence of CR-GNB of all patients admitted for 4 months.

\section{Results}

There are 1385 patients with positive CR-GNB cultures and 1535 negative patients in this study. Forty-five variables have statistical significant differences. We include the 17 variables in the multivariate logistic regression model and build three machine learning models for all variables. In terms of accuracy and the area under the receiver operating characteristic (AUROC) curve, the random forest is better than XGBoost and multivariate logistic regression model, and better than decision tree model (accuracy: $84 \%>82 \%>81 \%>72 \%$ ), (AUROC: $0.9089>0.8947 \approx 0.8987>0.7845$ ). In the 4 -month prospective study, 81 cases were predicted to be positive in CR-GNB culture within 7 days, 146 cases were predicted to be negative, 86 cases were positive, and 120 cases were negative, with an overall accuracy of $84 \%$ and AUROC of $91.98 \%$.

\section{Conclusions}

Prediction models by machine learning can predict the occurrence of CR-GNB colonization or infection within a week period, and can real-time predict and guide medical staff to identify high-risk groups more accurately.

\section{Background:}

Multidrug-resistant bacteria (MDRB) infection is a global healthcare crisis which not only increases the length and cost of hospitalization but also advances the use of novel antibiotics ${ }^{1}$. This is a vicious loop in which novel antibiotics create selective pressure on bacteria and then induce the occurrence of $\mathrm{MDRB}^{2,3}$. In recent years, the main MDRBs are carbapenem resistance gram-negative bacteria (CR-GNB), including carbapenem-resistant Enterobacteriaceae, carbapenem-resistant Acinetobacter baumannii, and carbapenem-resistant Pseudomonas aeruginosa, which are on the list of priority pathogens by the World Health Organization ${ }^{1}$. The prevention and control of CR-GNB is the difficulty and focus for clinicians in the intensive care unit (ICU). Clinicians take many measures to prevent the occurrence of CR-GNB, which also reflects the dilemma from the side ${ }^{4-7}$. The staff's compliance and CR-GNB unknown transmission limit the prevention and control measures.

The prevention and control of CR-GNB are mainly carried out by cutting off the route of transmission and protecting the susceptible population. The CRGNB transmission route is difficult to monitor in daily clinical work and identification of high-risk populations is easier comparatively. Many studies focus on the high-risk factors of CR-GNB colonization and infection included carbapenem antibiotic exposure, third-generation cephalosporins exposure, invasive manipulation, mechanical ventilation and so on ${ }^{8-10}$. Targeted prevention and control measures for patients with high-risk factors seem to have good potential, but no effective tools have been developed to predict the occurrence of drug-resistant bacteria.

In the past, the multivariate logistic regression is usually used to establish clinical prediction models. However, there are many new ideas to solve the problem with the upsurge of machine learning ${ }^{11,12}$. In the field of intensive care medicine, machine learning has been successfully applied in the classification and prediction of the mortality of sepsis, acute kidney injury and the mortality in ICU hospital, which is more stable and efficient than the traditional modeling ${ }^{13-16}$. At present, the prediction models using machine learning is more accurate and competitive than the traditional multivariate logistic regression model due to the advantages of large samples and novel algorithms. It is unknown whether machine learning has the same effect in the prediction of CR-GNB.

In this study, we establish a CR-GNB prediction model using our center database by machine learning with the purpose of recognition whether they were colonized or infected with CR-GNB in a week. We also have verified the accuracy of the prediction model through a small prospective study.

\section{Methods}

Study Characteristics

This study is a retrospective control study in the model establishment and prospective study in the model validation. The second affiliated hospital of Zhejiang university school of medicine is an academic teaching hospital. The general ICU has 40 monitoring beds and treats 2000 patients every year 
with a full range of diseases. The general ICU database uses MIMIC database structure to collect basic patients' information, medical advice, image examination, laboratory testing, nursing and doctor documents. The database is comprised of nearly 10,000 ICU patients, and the data have been updated daily.

\section{Model Establishment}

1) Participants: all the patients in the general ICU database. Patients with positive culture of CR-GNB is defined as CR-GNB carriage. Exclude standard: i) patients with hospital stay less than a week; ii) patients with a positive culture of CR-GNB within 48 hours of admission; iii) patients with only one positive culture result and considering as contamination during hospitalization. See Fig. 1 for the flow chart.

2) Variable selection: demographic data, vital signs, basic and primary diseases, important test indicators, operations histories, and antibiotic use records in the prior month. The time range of variable collection was determined according to the positive CR-GNB culture time. i) patients with positive CR-GNB culture within a week of admission, the variable collection period is limited in 48 hours of admission. ii) patients with positive culture more than a week, the collection period is limited between admission to a week before the positive time. iii) according to the previous investigation, $87 \%$ of patients would carry CR-GNB within 2 weeks at admission, so the variable collection period of negative patients is within 1 week of admission. We select 65 variables into our study, as detailed in Table 1. 
Table 1

Basic characteristics of variables

\begin{tabular}{|c|c|c|c|}
\hline & Positive & Negative & $\mathbf{P}$ \\
\hline $\mathrm{N}$ & 1385 & 1535 & \\
\hline AGE & 58.27 & 58.60 & 0.63 \\
\hline Gender (Man) & $60.20 \%$ & $62.21 \%$ & 0.000 \\
\hline Weight & 68.32 & 57.97 & 0.081 \\
\hline Single & 703 & 875 & 0.001 \\
\hline Hospital residence history (< a month) & 135 & 256 & 0.000 \\
\hline Primary disease & 1028 & 1171 & 0.087 \\
\hline Infectious Diseases & 163 & 194 & / \\
\hline Trauma & 369 & 305 & / \\
\hline Cardio-cerebrovascular accident & 247 & 255 & / \\
\hline Postoperative diseases & 151 & 227 & / \\
\hline Internal medicine diseases & 98 & 187 & / \\
\hline Other & 357 & 364 & / \\
\hline Malignant tumor & 102 & 150 & 0.034 \\
\hline Chronic kidney disease & 71 & 101 & 0.134 \\
\hline Diabetes Mellitus & 108 & 114 & 0.550 \\
\hline Hematological Disease & 62 & 65 & 0.630 \\
\hline Cardiovascular disease & 52 & 66 & 0.549 \\
\hline Liver cirrhosis & 7 & 21 & 0.02 \\
\hline Chronic lung disease & 7 & 12 & 0.385 \\
\hline CRRT & 144 & 214 & 0.04 \\
\hline Mechanical ventilation & 836 & 1238 & 0.000 \\
\hline Tracheostomy & 127 & 143 & 0.892 \\
\hline Invasive catheterization & 343 & 674 & 0.000 \\
\hline History of cephalosporins (< a month) & 450 & 906 & 0.000 \\
\hline History of carbapenems (< a month) & 388 & 524 & 0.000 \\
\hline History of glucocorticoids (< a month) & 310 & 400 & 0.021 \\
\hline Operation history (< six month) & 386 & 791 & 0.000 \\
\hline Drainage tube & 533 & 576 & 0.594 \\
\hline Fever (temperature $>38.5^{\circ} \mathrm{C}$ ) & 679 & 777 & 0.390 \\
\hline High APACHE II scores (> 20 points) & 594 & 689 & 0.277 \\
\hline APACHE II scores & 18.76 & 20.32 & 0.000 \\
\hline Charlson scores & 2.18 & 2.28 & 0.180 \\
\hline Hypoleukaemia & 97 & 186 & 0.000 \\
\hline Thrombopenia & 176 & 257 & 0.003 \\
\hline
\end{tabular}

CRRT: Continuous Renal Replacement Therapy; GCS : Glasgow coma scale; SBP: Systolic pressure; WBC: White blood cell count; NEP: neutrophil percentage; HCT: Hematokrit; CRP: C-Reactive Protein; PCT: procalcitonin; PLT: Platelet count; HB: hemoglobin; CR: Creatinine; CTnl: troponin; CK-MB: Creatine kinase isoenzyme MB; ALT: Alanine transaminase; AST: Aspartate aminotransferase; TBIL: Total bilirubin; DBIL: Direct bilirubin; IBIL: Indirect bilirubin; TP: Total protein; ALB: Albumin ; PT: Prothrombin time; APTT: Activated partial thromboplastin time; FBG: Fibrinogen; GLU: Blood glucose; LAC: Lactic acid;

The maximum and minimum values of the subscript of some parameters are the maximum or minimum values in the specified collection node, which are determined according to the clinical significance. 


\begin{tabular}{|c|c|c|c|}
\hline & Positive & Negative & $\mathbf{P}$ \\
\hline Acute kidney injury & 301 & 424 & 0.001 \\
\hline Acute liver failure & 148 & 255 & 0.000 \\
\hline Pulse $_{\max }$ & 109.11 & 115.79 & 0.000 \\
\hline Respiratory rate $_{\max }$ & 24.58 & 27.03 & 0.000 \\
\hline Temperature $_{\max }$ & 38.33 & 38.47 & 0.000 \\
\hline $\mathrm{GCS}_{\min }$ & 8.38 & 10.64 & 0.000 \\
\hline $\mathrm{SBP}_{\max }$ & 166.16 & 170.75 & 0.000 \\
\hline $\mathrm{WBC}_{\max }$ & 14.427 & 16.46 & 0.000 \\
\hline $\mathrm{WBC}_{\min }$ & 8.67 & 7.23 & 0.000 \\
\hline $\mathrm{NEU}_{\max }$ & 89.58 & 91.51 & 0.000 \\
\hline $\mathrm{HCT}_{\max }$ & 44.04 & 48.02 & 0.009 \\
\hline $\mathrm{CRP}_{\max }$ & 118.32 & 132.79 & 0.000 \\
\hline $\mathrm{PCT}_{\max }$ & 5.51 & 7.36 & 0.000 \\
\hline $\mathrm{PLT}_{\min }$ & 137.96 & 114.23 & 0.000 \\
\hline $\mathrm{HB}_{\min }$ & 78.06 & 73.66 & 0.000 \\
\hline $\mathrm{CR}_{\max }$ & 104.40 & 131.68 & 0.000 \\
\hline $\mathrm{CTnI}_{\max }$ & 3.73 & 12.00 & 0.086 \\
\hline $\mathrm{CK}^{-\mathrm{MB}_{\max }}$ & 42.61 & 62.94 & 0.000 \\
\hline $\mathrm{ALT}_{\max }$ & 99.73 & 148.24 & 0.003 \\
\hline $\mathrm{AST}_{\max }$ & 197.71 & 354.71 & 0.001 \\
\hline $\mathrm{TBIL}_{\max }$ & 27.07 & 35.63 & 0.000 \\
\hline $\mathrm{DBIL}_{\max }$ & 8.78 & 12.44 & 0.000 \\
\hline $\mid \mathrm{BIL} \mathrm{L}_{\max }$ & 18.98 & 24.66 & 0.000 \\
\hline $\mathrm{TP}_{\max }$ & 61.96 & 65.71 & 0.000 \\
\hline $\mathrm{ALB}_{\min }$ & 28.59 & 26.58 & 0.000 \\
\hline PTmax & 17.44 & 18.80 & 0.000 \\
\hline $\mathrm{APTT}_{\max }$ & 51.78 & 57.47 & 0.000 \\
\hline $\mathrm{FBG}_{\max }$ & 4.94 & 5.39 & 0.000 \\
\hline D-Dimer ${ }_{\max }$ & 7043.89 & 8052.42 & 0.000 \\
\hline $\mathrm{GLU}_{\max }$ & 12.72 & 14.49 & 0.001 \\
\hline $\mathrm{LAC}_{\max }$ & 3.41 & 4.39 & 0.000 \\
\hline \multicolumn{4}{|c|}{$\begin{array}{l}\text { CRRT: Continuous Renal Replacement Therapy; GCS : Glasgow coma scale; SBP: Systolic pressure; WBC: White blood cell count; NEP: neutrophil } \\
\text { percentage; HCT: Hematokrit; CRP: C-Reactive Protein; PCT: procalcitonin; PLT: Platelet count; HB: hemoglobin; CR: Creatinine; CTnl: troponin; CK-MB: } \\
\text { Creatine kinase isoenzyme MB; ALT: Alanine transaminase; AST: Aspartate aminotransferase; TBIL: Total bilirubin; DBIL: Direct bilirubin; IBIL: Indirect } \\
\text { bilirubin; TP: Total protein; ALB: Albumin ; PT: Prothrombin time; APTT: Activated partial thromboplastin time; FBG: Fibrinogen; GLU: Blood glucose; } \\
\text { LAC: Lactic acid; }\end{array}$} \\
\hline \multicolumn{4}{|c|}{$\begin{array}{l}\text { The maximum and minimum values of the subscript of some parameters are the maximum or minimum values in the specified collection node, } \\
\text { which are determined according to the clinical significance. }\end{array}$} \\
\hline
\end{tabular}


3) Statistical methods: we use MySQL and Navicat to complete data collection with structured query language. Data analysis are accomlished through R and Rstudio with related packets. Multivariate logistic regression and three machine learning algorithms, as decision tree, random forest, and XGBoost, are selected to establish the models. The main R packets include "glm", "rpart", "randomForest", "xgboost" and "rattle". We delete independent variables with the missing more than $50 \%$ and the other missing values are processed by multiple interpolations. In univariate analysis, numerical variables are tested by independent sample T-test, dichotomous variables are completed by the chi-square test, and a P value less than 0.05 is considered as statistical significance. All samples are grouped into $70 \%$ training set, $15 \%$ validation set, and $15 \%$ test set. Multivariate logistic regression uses the step-by-step decreasing method to adjust the parameters. Five hundred trees are constructed and the exhaustive method is used to adjust the parameters in the Random forest, and the importance of measurable variables is presented by the corresponding visual package. The specific and task parameters of the XGBoost linear rise are adjusted according to the performance of the model. The evaluation parameters include sensitivity, specificity, positive predictive value, negative predictive value, and area under the receiver operating characteristic (AUROC) curves.

Prospective Study

1) Study duration: 09/01/2019 to $12 / 31 / 2019$.

2) Protocol: i) determination of the optimal prediction model through model evaluation; ii) application of the model for all patients admitted to ICU to make daily predictions. iii) termination of prediction with the following conditiions: a) when prediction results suggest high risk (CR-GNB carriage will positive within 7 days), stop prediction and enter the 7-day observation period; b) when patients leave the ICU ward, including transfer, discharge or death. iv) analysis of the CR-GNB carriage within 7 days compare with the predicted results and calculation of the relevant evaluation indexes and evaluation the model performance. v) all the predicted results are kept secret to clinicians, and this study does not interfere with clinical decision-making.

\section{Results:}

Prediction models

A total of 3303 patients are included in the study, of which 1768 patients are culture positive. According to the exclusion criteria, we include 1385 CR-GNB carriage patients and 1535 negative patients. Among the positive CR-GNB carriages, carbapenem-resistant Acinetobacter baumannii accounts for $59.16 \%$, carbapenem-resistant Klebsiella pneumoniae accounts for $21.21 \%$, and carbapenem-resistant Pseudomonas aeruginosa accounts for $18.25 \%$. In terms of the distribution of cultural sites, sputum culture accounts for $80.95 \%$. As far as the types of diseases, the center is dominated by multiple injuries, especially severe craniocerebral trauma, followed by various infectious diseases, and cardio-cerebrovascular accidents. See Table 5 for details.

In univariate analysis, there are 45 variables with statistical differences as Table 1 . In the multivariate logistic regression model, a stable model is obtained by incorporating 17 variables, see as Table 2 . These risk factors includes gender, invasive catheterization, mechanical ventilation and hospital residence history over the past month, vital signs including systolic blood pressure, respiratory rate and glasgow coma scale, laboratory indicators including white blood cell count, hematocrit, C-reaction protein, direct bilirubin, total protein, and fibrinogen. The use of cephalosporins and carbapenems is also a high-risk factor. In the models established by three common machine learning methods, random forest presents the important characteristics of model parameters, as Fig. 2, in which hospital residence history over the past month, total protein and respiratory rate are the main risk factors which have much overlap with the multivariate logistic regression model. 
Table 2

Parameters in the multivariate logistic regression model

\begin{tabular}{|ll|}
\hline Parameters & P \\
\hline Gender & 0.000 \\
\hline Invasive catheterization & 0.000 \\
\hline Drainage tube & 0.006 \\
\hline Mechanical ventilation & 0.000 \\
\hline Hospital residence history (<a month) & 0.000 \\
\hline History of cephalosporins (<a month) & 0.000 \\
\hline Hypoleukaemia & 0.000 \\
\hline Respiratory rate max $_{\text {max }}$ & 0.000 \\
\hline $\mathrm{GCS}_{\text {min }}$ & 0.000 \\
\hline $\mathrm{SBP}_{\max }$ & 0.014 \\
\hline $\mathrm{APACHE} \mathrm{Il}$ scores & 0.000 \\
\hline $\mathrm{WBC}_{\max }$ & 0.000 \\
\hline $\mathrm{HCT}_{\max }$ & 0.003 \\
\hline $\mathrm{CRP}_{\max }$ & 0.005 \\
\hline $\mathrm{DBIL}_{\max }$ & 0.000 \\
\hline $\mathrm{TP}_{\max }$ & 0.000 \\
\hline $\mathrm{FBG}_{\text {max }}$ & 0.005 \\
\hline
\end{tabular}

The multivariate logistic regression model is well fitted and has high prediction accuracy ( $84 \%$ in validation and $81 \%$ in test). This traditional model has great specificity $(91.27 \%$ in validation and $91.73 \%$ in test) and a positive predictive value $(87.21 \%$ in validation and $85.14 \%$ in test). Except for the decision tree model, the fitting effect of random forest and XGBoost are similar, the sensitivity of XGBoost (77.23\%in validation and $74.47 \%$ in test) is similar with that of random forest $(70.30 \%$ in validation and $77.66 \%$ in test) and the specificity of random forest is the best ( $92.86 \% \%$ in validation and $87.97 \%$ in test). In terms of accuracy and AUROC curve, random forest (0.9182 in validation and 0.9089 in test) is better than XGBoost (0.9127 in validation and 0.8947 in test), and multivariate logistic regression model ( 0.9073 in validation and 0.8983 in test), and is generally better than decision tree model (0.8178 in validation and 0.7845 in test), see as Table 3, Fig. 3 and Fig. 4.

Table 3

Evaluation Result of machine learning and multiple logistic regression model validation set and test set

\begin{tabular}{|c|c|c|c|c|c|c|c|c|c|c|c|c|}
\hline & \multicolumn{4}{|c|}{ Validation set } & \multicolumn{3}{|c|}{ Test set } & \multirow[b]{2}{*}{ specificity } & \multirow[b]{2}{*}{ PPV } & \multirow[b]{2}{*}{ NPV } & \multirow[b]{2}{*}{ Accuracy } & \multirow[b]{2}{*}{ AUROC } \\
\hline & Sensitivity & specificity & PPV & NPV & Accuracy & AUROC & Sensitivity & & & & & \\
\hline $\begin{array}{l}\text { Decision } \\
\text { tree }\end{array}$ & 67.60 & 81.78 & 77.84 & 72.73 & 0.75 & 0.8178 & 62.86 & 80.35 & 74.58 & 70.23 & 0.72 & 0.7845 \\
\hline $\begin{array}{l}\text { Random } \\
\text { forest }\end{array}$ & 70.30 & 92.86 & 88.75 & 79.59 & 0.83 & 0.9182 & 77.66 & 87.97 & 82.02 & 84.78 & 0.84 & 0.9089 \\
\hline XGBoost & 77.23 & 89.68 & 85.71 & 83.09 & 0.84 & 0.9127 & 74.47 & 87.97 & 81.40 & 82.98 & 0.82 & 0.8947 \\
\hline $\begin{array}{l}\text { multivariate } \\
\text { logistic } \\
\text { regression }\end{array}$ & 74.26 & 91.27 & 87.21 & 81.56 & 0.84 & 0.9073 & 67.02 & 91.73 & 85.14 & 79.74 & 0.81 & 0.8983 \\
\hline
\end{tabular}

According to the effect of the model, we finally choose the random forest as the optimal prediction model for the prospective study.

Prospective Study

In the 4-month prospective study, a total of 673 patients are treated in our center, and the total number of prediction period by the model is 4553 . Among them, 431 patients with hospitalization days less than 7 days and 36 patients with positive in 48 hours as admission are excluded. It is finally predicted that 81 cases are positive and 146 cases are negative. There are 86 positive cases and 120 negative cases after 7 days of observation. The sensitivity is 
$72.28 \%$, lower than the performance of the test set, but the specificity is as high as $93.65 \%$, better than that of the previous test set. Therefore our model is computationally efficient and achieves high accuracy of $84 \%$ and AUROC of $91.98 \%$. it is equivalent to the accuracy of the validation set and the test set. In the verification stage, the proportion of sputum samples was still the largest, and the proportion of Pseudomonas aeruginosa was the highest, more detail can be seen in Tables 4 and 5 .

Table 4

Prospective study results

\begin{tabular}{|c|c|c|c|c|c|c|}
\hline \multicolumn{3}{|l|}{ Duration } & \multicolumn{4}{|c|}{ 2019.09.01-2019.12.31 } \\
\hline \multicolumn{3}{|l|}{ Total patients } & \multicolumn{4}{|l|}{673} \\
\hline \multicolumn{3}{|c|}{ Total number of predictions } & \multicolumn{4}{|l|}{4553} \\
\hline \multicolumn{3}{|c|}{ Number of patients hospitalized $<7$ days } & \multicolumn{4}{|l|}{431} \\
\hline \multicolumn{3}{|c|}{ Numbers of positive active surveillance patients at admission } & \multicolumn{4}{|l|}{36} \\
\hline \multicolumn{3}{|c|}{ Predictive positive patients } & \multicolumn{4}{|l|}{81} \\
\hline \multicolumn{3}{|c|}{ Predictive negative patients } & \multicolumn{4}{|l|}{146} \\
\hline \multicolumn{3}{|c|}{ Actual positive patient } & \multicolumn{4}{|l|}{86} \\
\hline \multicolumn{3}{|c|}{ Actual negative patient } & \multicolumn{4}{|l|}{120} \\
\hline & Sensitivity & specificity & PPV & NPV & Accuracy & AUROC \\
\hline Evaluation Result & 72.28 & 93.65 & 90.12 & 80.82 & 0.84 & 0.9198 \\
\hline
\end{tabular}

Table 5

The positive culture sites and bacterial distribution

\begin{tabular}{|c|c|c|c|}
\hline & & retrospective control study & Prospective study \\
\hline Total cases & & 1385 & 86 \\
\hline \multirow[t]{4}{*}{ Bacterial distribution } & CRAB & $820(59.16 \%)$ & $26(30.23 \%)$ \\
\hline & CRKP & $294(21.21 \%)$ & $26(30.23 \%)$ \\
\hline & CRPA & $253(18.25 \%)$ & $31(36.05 \%)$ \\
\hline & Other CRE & $22(1.59 \%)$ & $3(3.49 \%)$ \\
\hline \multirow[t]{5}{*}{ Sites distribution } & Sputum & 1122(80.95\%) & 73(84.88\%) \\
\hline & Blood & $97(7.00 \%)$ & 0 \\
\hline & Stool & $49(3.54 \%)$ & $9(10.47 \%)$ \\
\hline & Urine & $40(2.89 \%)$ & 0 \\
\hline & Others & $77(5.56 \%)$ & $4(4.65 \%)$ \\
\hline
\end{tabular}

\section{Discussion:}

In this study, we build the prediction model of CR-GNB carriages within a week at admission by machine learning. We analyze to further verify the accuracy of the model through a 4-month prospective and consecutive predictions.

With the worldwide spread of CR-GNB in ICU, clinicians invest a lot of times and resources in nosocomial prevention and control measures, including colonization supervision, contact isolation, hand hygiene, antibiotic control and so on ${ }^{2,6}$. However, in the face of heavy clinical work, the implementation rate of these measures has been criticized. The normalization of nosocomial prevention and control measures is not only the compliance of medical staff but also the problem of cost and benefits. Control the source of infection, cut off the route of transmission, and protect the vulnerable population are three classical pathways. Current research has found more and more dormant sources of infection, including fiberoptic bronchoscope, ICU flume ${ }^{17,18}$. The route of transmission also has more possibility of analysis with the assistance of next-generation sequencing ${ }^{19}$. Many pieces of researches focus on Protecting susceptible people because of its simplicity and effectiveness ${ }^{5,20,21}$. Some studies pay attention to the identification of high-risk factors, which are determined by building models ${ }^{21-24}$. Thomas and his colleagues predicted the infection of MDR by multivariate logical regression model by the public database and found that the main risk factors of MDR infection were the high use of antibiotics previously, the site and degree of infection in 
the previous three months ${ }^{25}$. The researchers included a total of 120,000 samples, but there were a few variables and the models could only vaguely indicate which patients were likely to develop into the infections. Katherine and his teams used a multiple logical regression model and a decision tree model to assess the risk of extended Spectyum- $\beta$ lactamase (ESBLs) bacteremia through the data of 1288 cases of gram-negative bacteremia. A total of 14 variables were included. The prediction effect of the decision tree and multivariate logistic regression model for predicting ESBL infection was similar $^{21}$. However, the research sample size and variables are limited, and the incoordination between the number of positive cases (15\%) and that of control cases (85\%) restricted the performance of machine learning. Wang et al used 512 patients to predict MDR carriages and found that males, high CRP levels, and high Pitt scores were high-risk predictors, and a line chart was used to predict the occurrence of MDR ${ }^{24}$. In our study, invasive procedures include endotracheal intubation, intravenous intubation, drainage tube, and the hospital residence history over the past month are high-risk factors for CRGNB carriages, which have been identified in other studies ${ }^{9,22}$. According to the history of residence in other hospitals, our center adopts preemptive isolation and active surveillance which can reduce the incidence of carbapenem-resistant Enterobacteriaceae (CRE) ${ }^{26}$. However, these kinds of literatures only provide information on which patients may develop MDR colonization or infection, but the exact time is unknown. As a result, the prevention and control of nosocomial infection measures are faced with the problems of when to implement and when to remove, as well as the cost-effectiveness, clinical burnout. Therefore, our center developed a CR-GNB prediction model in a week for ICU patients, which aims to carry out more targeted prevention including single isolation in advance, special management, and other measures. At present, this study has completed the verification of clinical applicability, and the subsequent clinical randomized controlled trial will be conducted to verify its clinical practicability.

We set one week as the forecast period in several aspects. First of all, too short or too long prediction periods will affect the performance of the prediction model. Secondly, ICU hospitalization as a high-risk factor for CR-GNB, the longer the ICU hospital stay, the higher the incidence of CR-GNB. According to the average hospitalization days, about 6 days in our center, most of the patients with hospitalization in a week are postoperative patients who are not the beneficiaries of this study. Lastly, according to the pre-experimental results, the peak time of CR-GNB carriages in our center was 5 days, then decreased slowly. One week as the prediction node can balance and the comparability in the trial group and the control group. Besides, sputum samples were still dominant in the colonization and infection of CR-GNB in this study, which was related to the types of diseases, including severe craniocerebral trauma and community-acquired pneumonia, which required long-term mechanical ventilatio. and the proportion of. These patients sent sputum samples for examination are higher than that of other parts and stay longer than the general postoperative patient trial. Therefore they are more likely to be included and caused bias in the study.

This study uses the central database, which has both advantages and disadvantages. On the one hand, the local database has with the diversified and integrated variables, which is difficult to achieve in the public database. The central database has been updated in real-time and provides the feasibility for continuous prediction. Also, the real-time updated database can continuously carry out iterative learning for the model to incorporate data and keep pace with the times. On the other hand, the public database has more data and complete diseases than the single-center database, based by stable models and multi-center researches. However, the problem is also very prominent. The variables are fixed and may simplify the number of variables to ensure the integrity, followed by a slow update and unable to achieve timely prediction. The number adopted in this study is limited, and as single-center research, follow-up promotion will take a long time.

\section{Conclusion:}

The prediction model by machine learning can predict the occurrence of CR-GNB carriage within one week, with a success rate of $84 \%$. This model can predict the high-risk groups of CR-GNB carriers in real-time and help guide medical staff to take more targeted prevention and control of nosocomial measures.

\section{Abbreviations}

\section{CR-GNB}

carbapenem-resistance gram-negative bacteria;

AUROC

area under the receiver operating characteristic;

MDRB

multidrug-resistant bacteria;

ICU

intensive care unit

\section{Declarations}

\section{Ethics approval and consent to participate:}

Our research has been approved by the ethics committee of the second affiliated hospital of Zhejiang University School of Medicine.

Consent for publication: 


\section{Availability of data and materials:}

The datasets used and/or analysed during the current study are available from the corresponding author on reasonable request.

\section{Competing interest:}

None.

\section{Funding:}

No funding

\section{Author contributions:}

Qiqiang Liang write manuscript and analyze data; Qinyu Zhao assist at data cleaning and modeling; Xin Xu assist at data secondary audit, independent variable screening, and chart drawing; Yu Zhou and Jie Xu establish the database and collect original data. Huang Man corrects the data of the paper;

\section{Acknowledgements:}

Not applicable.

\section{References}

1. Willyard C. The drug-resistant bacteria that pose the greatest health threats. Nature 2017; 543: 15. 2017/03/03. DOI: 10.1038/nature.2017.21550.

2. Martin-Loeches I, Leone M, Madach K, et al. Antibiotic therapy in the critically ill - expert opinion of the Intensive Care Medicine Scientific Subcommittee of the European Society of Anaesthesiology. European journal of anaesthesiology 2017; 34: 215-220. DOI: 10.1097/EJA.0000000000000595.

3. Zilberberg MD, Nathanson BH, Sulham K, et al. Carbapenem resistance, inappropriate empiric treatment and outcomes among patients hospitalized with Enterobacteriaceae urinary tract infection, pneumonia and sepsis. BMC infectious diseases 2017; 17: 279. 2017/04/19. DOI: 10.1186/s12879017-2383-z.

4. Palmore TN and Henderson DK. Managing transmission of carbapenem-resistant enterobacteriaceae in healthcare settings: a view from the trenches. Clinical infectious diseases : an official publication of the Infectious Diseases Society of America 2013; 57: 1593-1599. 2013/08/13. DOI: 10.1093/cid/cit531.

5. Karampatakis T, Tsergouli K, losifidis E, et al. Impact of active surveillance and infection control measures on carbapenem-resistant Gram-negative bacterial colonization and infections in intensive care. J Hosp Infect 2018; 99: 396-404. Article. DOI: 10.1016/j.jhin.2018.05.010.

6. Gandra S and Ellison RT, 3rd. Modern trends in infection control practices in intensive care units. Journal of intensive care medicine 2014; 29: 311326. 2013/06/12. DOI: 10.1177/0885066613485215.

7. Asensio A, Cantero M, Shaw E, et al. Control strategies for carbapenemase-producing Enterobacteriaceae at different levels of the healthcare system. Enfermedades infecciosas y microbiologia clinica 2014; 32 Suppl 4: 61-66. 2014/12/30. DOI: 10.1016/s0213-005x(14)70176-4.

8. Frencken JF, Wittekamp BHJ, Plantinga NL, et al. Associations Between Enteral Colonization With Gram-Negative Bacteria and Intensive Care UnitAcquired Infections and Colonization of the Respiratory Tract. Clinical infectious diseases : an official publication of the Infectious Diseases Society of America 2017 2017/12/01. DOI: 10.1093/cid/cix824.

9. Giacobbe DR, Del Bono V, Bruzzi P, et al. Previous bloodstream infections due to other pathogens as predictors of carbapenem-resistant Klebsiella pneumoniae bacteraemia in colonized patients: results from a retrospective multicentre study. European journal of clinical microbiology \& infectious diseases : official publication of the European Society of Clinical Microbiology 2017; 36: 663-669. D0I: 10.1007/s10096-016-2843-1.

10. Gutierrez-Gutierrez B, Salamanca E, de Cueto M, et al. Effect of appropriate combination therapy on mortality of patients with bloodstream infections due to carbapenemase-producing Enterobacteriaceae (INCREMENT): a retrospective cohort study. Lancet Infectious Diseases 2017; 17: 726-734. DOI: 10.1016/S1473-3099(17)30228-1.

11. Komorowski M, Celi LA, Badawi O, et al. The Artificial Intelligence Clinician learns optimal treatment strategies for sepsis in intensive care. Nat Med 2018; 24: 1716-1720. 2018/10/24. DOI: 10.1038/s41591-018-0213-5.

12. Bailly S, Meyfroidt G and Timsit JF. What's new in ICU in 2050: big data and machine learning. Intensive Care Med 2018; 44: 1524-1527. 2017/12/28. DOI: 10.1007/s00134-017-5034-3.

13. Goodman KE, Simner PJ, Klein EY, et al. Predicting probability of perirectal colonization with carbapenem-resistant Enterobacteriaceae (CRE) and other carbapenem-resistant organisms (CROs) at hospital unit admission. Infect Control Hosp Epidemiol 2019; 40: 541-550. 2019/03/28. DOI: 10.1017/ice.2019.42. 
14. Huang $\mathrm{C}$, Murugiah $\mathrm{K}$, Mahajan S, et al. Enhancing the prediction of acute kidney injury risk after percutaneous coronary intervention using machine learning techniques: A retrospective cohort study. PLoS Med 2018; 15: e1002703. 2018/11/28. DOI: 10.1371/journal.pmed.1002703.

15. Meyer A, Zverinski D, Pfahringer B, et al. Machine learning for real-time prediction of complications in critical care: a retrospective study. The Lancet Respiratory Medicine 2018; 6: 905-914. DOI: 10.1016/s2213-2600(18)30300-x.

16. Nemati S, Holder A, Razmi F, et al. An Interpretable Machine Learning Model for Accurate Prediction of Sepsis in the ICU. Crit Care Med 2018; 46: 547553. 2017/12/30. DOI: 10.1097/CCM.0000000000002936.

17. Smith ZL, Dua A, Saeian K, et al. A Novel Protocol Obviates Endoscope Sampling for Carbapenem-Resistant Enterobacteriaceae: Experience of a Center with a Prior Outbreak. Dig Dis Sci 2017; 62: 3100-3109. Article. DOI: 10.1007/s10620-017-4669-9.

18. Smismans A, Ho E, Daniels D, et al. New environmental reservoir of CPE in hospitals.

19. Snitkin ES, Won S, Pirani A, et al. Integrated genomic and interfacility patient-transfer data reveal the transmission pathways of multidrug-resistant Klebsiella pneumoniae in a regional outbreak. Science translational medicine 2017; 9. DOI: 10.1126/scitranslmed.aan0093.

20. Magiorakos AP, Burns K, Rodríguez Baño J, et al. Infection prevention and control measures and tools for the prevention of entry of carbapenemresistant Enterobacteriaceae into healthcare settings: guidance from the European Centre for Disease Prevention and Control. Antimicrobial Resistance \& Infection Control 2017; 6. DOI: 10.1186/s13756-017-0259-z.

21. Goodman KE, Lessler J, Harris AD, et al. A methodological comparison of risk scores versus decision trees for predicting drug-resistant infections: $A$ case study using extended-spectrum beta-lactamase (ESBL) bacteremia. Infect Control Hosp Epidemio/ 2019; 40: 400-407. 2019/03/05. DOI: 10.1017/ice.2019.17.

22. Liu P, Li X, Luo M, et al. Risk Factors for Carbapenem-Resistant Klebsiella pneumoniae Infection: A Meta-Analysis. Microbial drug resistance 2018; 24 : 190-198. Article. DOI: 10.1089/mdr.2017.0061.

23. Maseda E, Salgado P, Anillo V, et al. Risk factors for colonization by carbapenemase-producing enterobacteria at admission to a Surgical ICU: A retrospective study. Enfermedades infecciosas y microbiologia clinica 2016 2016/03/27. DOI: 10.1016/j.eimc.2016.02.017.

24. Wang L, Huang X, Zhou J, et al. Predicting the occurrence of multidrug-resistant organism colonization or infection in ICU patients: development and validation of a novel multivariate prediction model. Antimicrobial Resistance \& Infection Control 2020; 9. D0I: 10.1186/s13756-020-00726-5.

25. Lodise TP, Bonine NG, Ye JM, et al. Development of a bedside tool to predict the probability of drug-resistant pathogens among hospitalized adult patients with gram-negative infections. BMC Infect Dis 2019; 19: 718. 2019/08/16. DOI: 10.1186/s12879-019-4363-y.

26. Liang Q, Yan C, Xu Z, et al. Preemptive isolation and active surveillance in the prevention and control of nosocomial infection reduce the incidence of carbapenem-resistant Enterobacteriaceae.

\section{Figures}




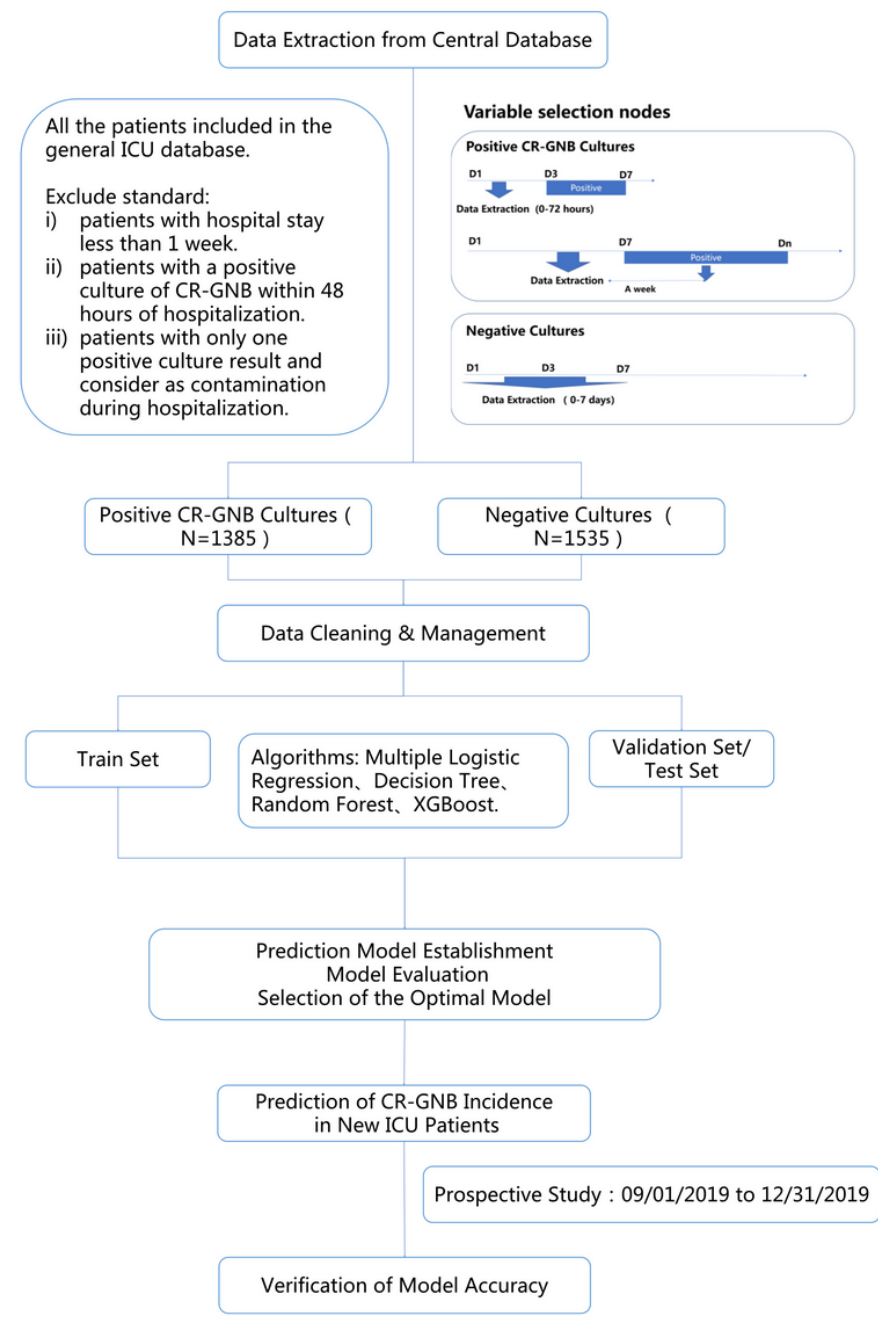

Figure 1

Flow chart of study. CR-GNB: carbapenem-resistance gram-negative bacteria; 


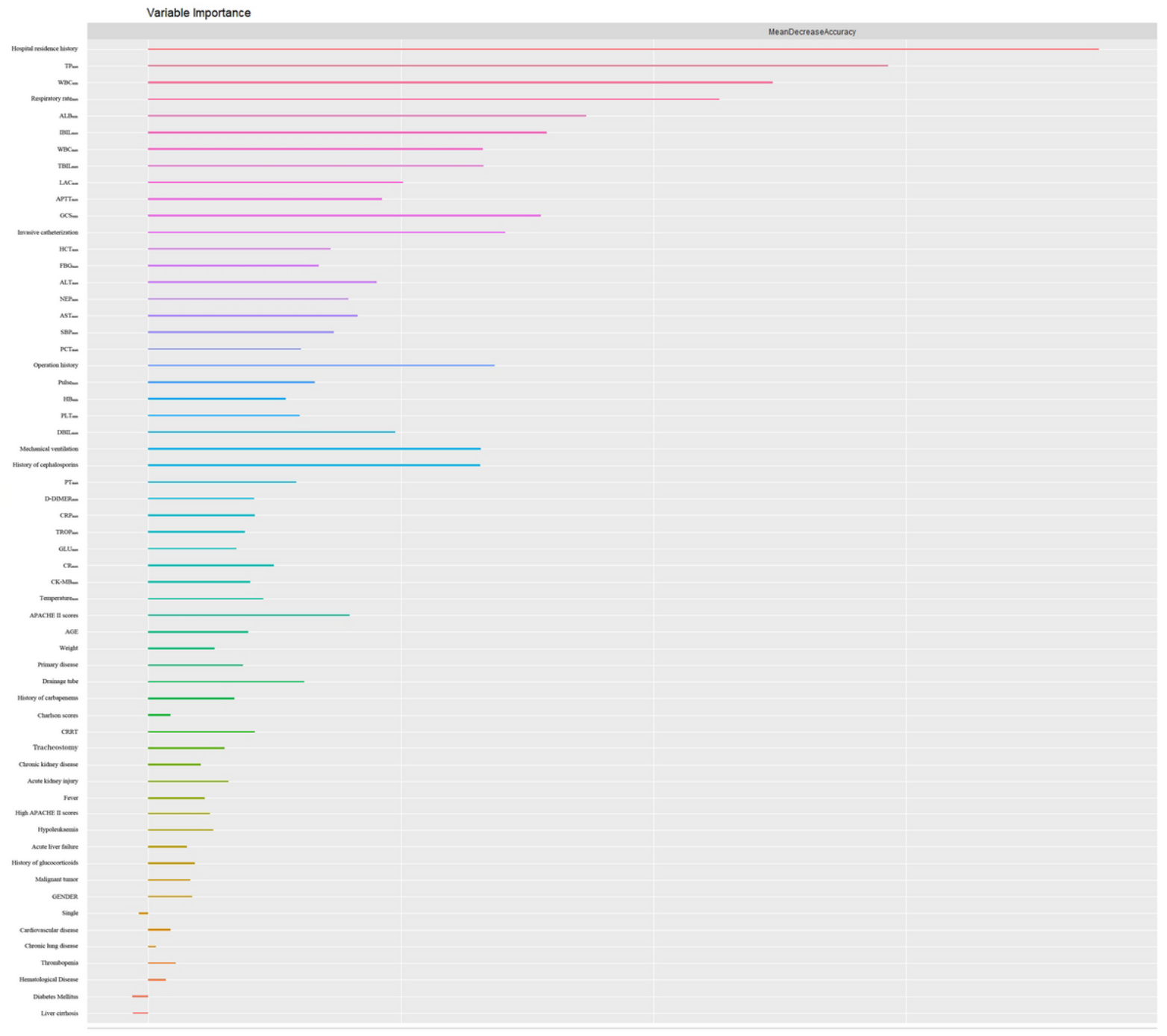

Figure 2

Important characteristics of model parameters by random forest included all variables. 


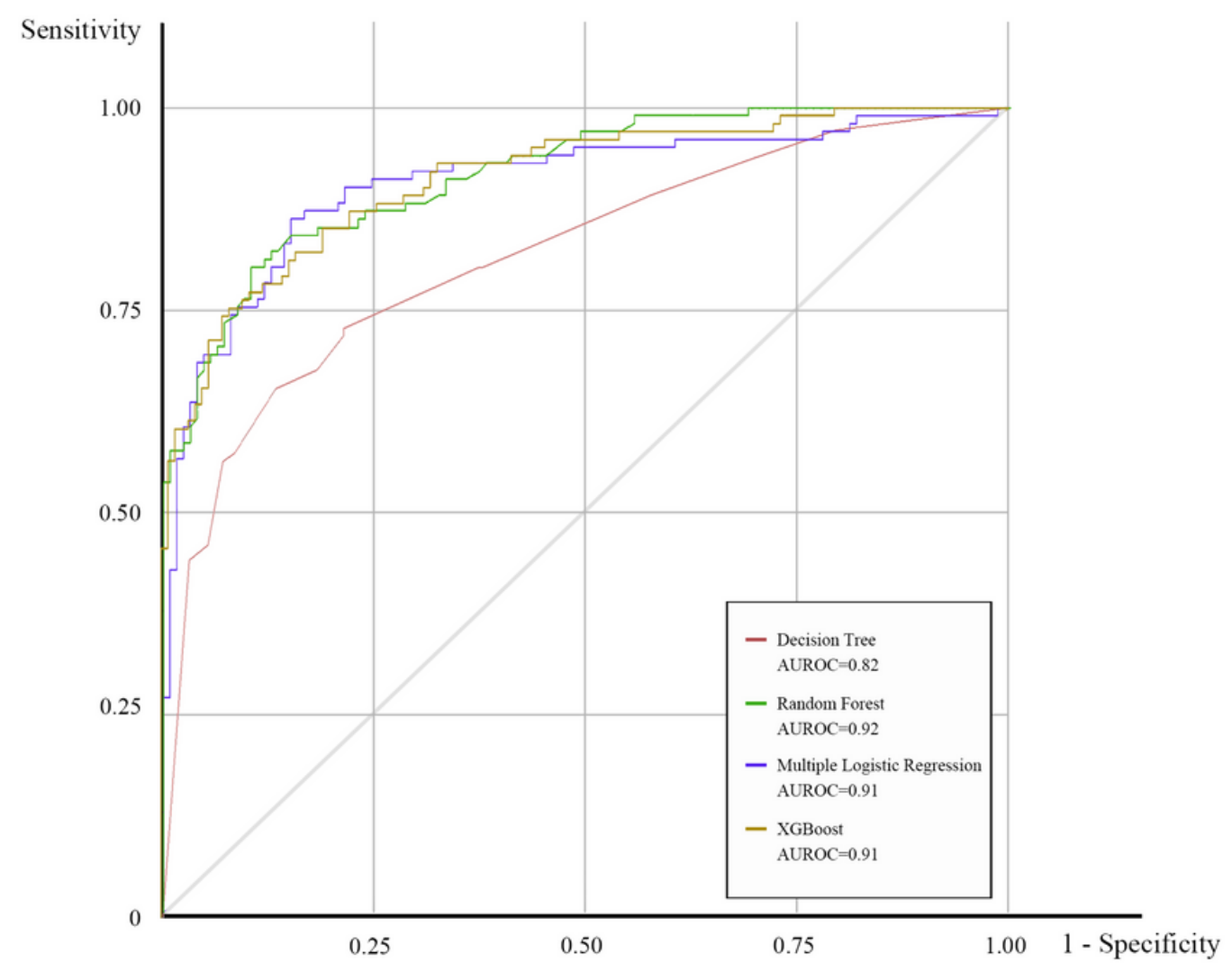

\section{Figure 3}

Area under the receiver operating characteristic curve of validation set for three machine learning and multivariate logistic regression. 


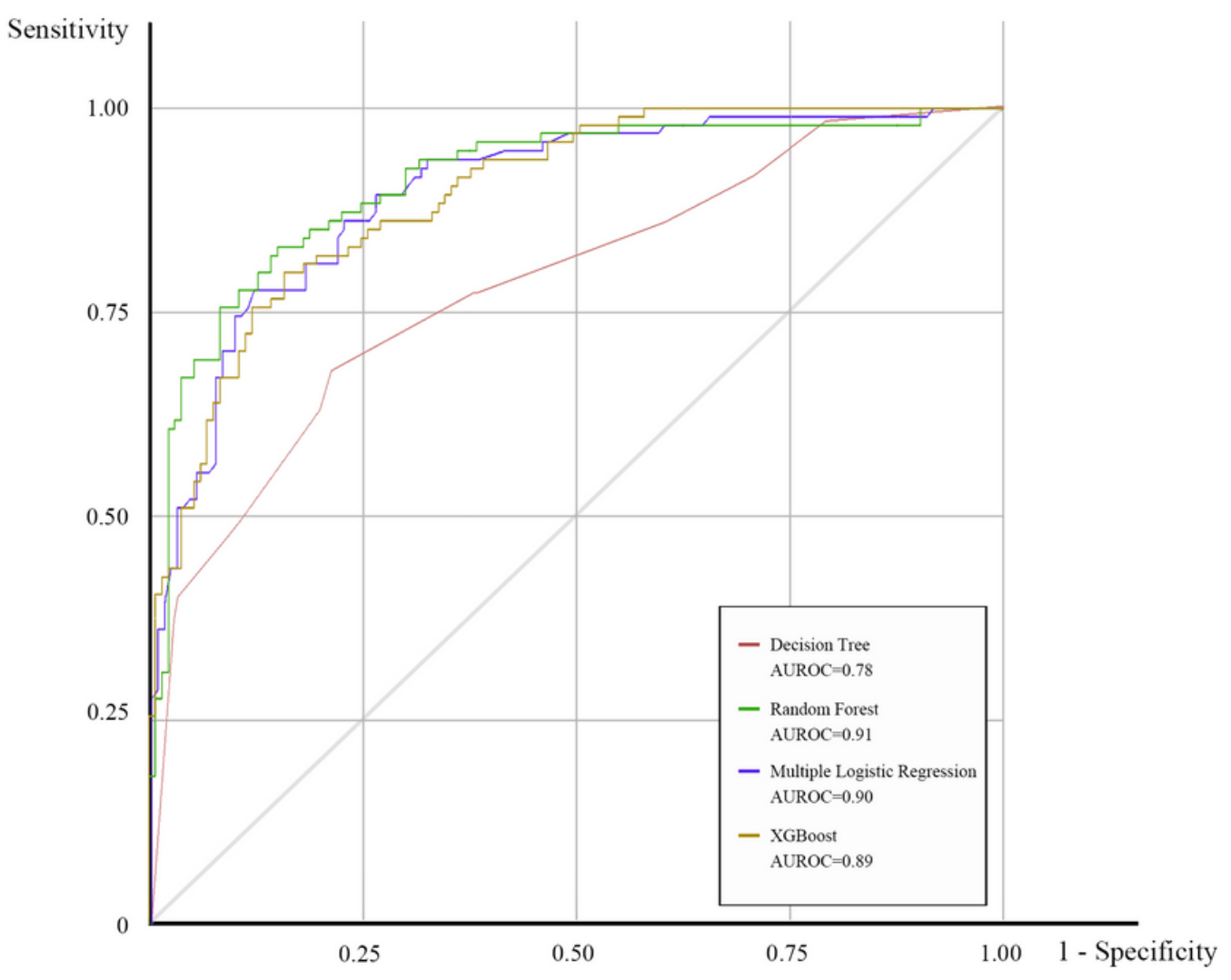

\section{Figure 4}

Area under the receiver operating characteristic curve of test set for three machine learning and multivariate logistic regression. 